\title{
Evolution of Quantification in Clinical Nuclear Medicine: A Brief Overview of Salient Uses and Upcoming Trends
}

\section{Arif Sheikh*}

Department of Radiology, Division of Nuclear Medicine, Molecular Imaging and Theranostics at Temple University, Lewis Katz School of Medicine, USA

"Corresponding author: Arif Sheikh, Professor and Co-director of Cardiac Nuclear Imaging, Department of Radiology, Division of Nuclear Medicine, Molecular Imaging and Theranostics at Temple University, Lewis Katz School of Medicine, USA, E-mail: Arif.Sheikh@tuhs.temple.edu

Received date: August 29, 2018; Accepted date: September 13, 2018; Published date: September 21, 2018

Copyright: @2018 Sheikh A. This is an open-access article distributed under the terms of the Creative Commons Attribution License, which permits unrestricted use, distribution, and reproduction in any medium, provided the original author and source are credited.

\begin{abstract}
Since the inception of Nuclear Medicine, the field strove to not only understand pathology through the qualitative evaluation of physiologic and molecular based processes, but also to quantify them to further differentiate normal from abnormal findings. Whereas the former has been well achieved and has evolved into the field of Molecular Imaging, the latter continues to evolve with a goal to provide a definite incremental improvement in diagnostic and clinical management of patients over that provided by the former alone. Quantification is found ubiquitously in Nuclear Medicine, such as in Renal, Thyroid, and Cardiac imaging, to therapies such as Thyroid Diseases and Y-90 microspheres (SIRT). This has also evolved with technology, starting from the simplest probes, static planar imaging, and cine planar and tomographic imaging, now extended to more absolute quantification with modern Positron Emission Tomography (PET-CT and PET-MR) machines, and further with the evolution of quantitative Single Photon Emission Computed Tomography (SPECT and SPECT-CT). This evolution is also a product of other developments in acquisition standardization, imaging methodologies, and understanding of radiotracer physiology. This article summarizes some of the more common procedures relying on quantification, and which direction the newer generation of quantitative based imaging developments promise to provide in the field.
\end{abstract}

Keywords: Nuclear medicine; Cardiac imaging; PET-CT; Radiotracer physiology

\section{Introduction}

\section{Imaging in general nuclear medicine}

Both relative and absolute quantification rely on some degree of understanding kinetics of the tracer being used. The simplest studies in Nuclear Medicine have used some form of relative quantitation. Relative split function of lung scans help guide extent of surgical resection. This study traditionally uses planar imaging and divide the lungs roughly by zones rather than the anatomical lobes. More recent studies have shown that 3-dimensional quantitation with SPECT and contouring the actual lobes instead of zones produces some differences in relative perfusions quantification. There is variable data whether an improvement with more accurate perfusion quantification with SPECT as opposed to planar imaging leads to improved outcomes, and is under continued study $[1,2]$.

Renal scintigraphy has used both relative and absolute quantitation in diagnosis and patient management. Relative cortical function of kidneys can assess whether a single kidney may be dysfunctional compared to the contralateral healthy one, whether due to obstruction, scarring, or other processes. Temporally evaluating parameters such as time to peak activity in-and subsequently half-time of clearance fromthe kidneys relate to the functionality of the cortices and degree of obstruction, respectively, allowing for the evaluation of the individual kidney. These rely less on the comparison to the contralateral one, and is a useful method when evaluating an individual kidney, such as a transplanted one. In fact, a basic estimate of absolute Glomerular Filtration Rate (GFR) can be made using imaging, provided the correct calibration of the camera is made, and the appropriate tracer specific corrections are used $[3,4]$; however, these parameters become limited once sub-acute to chronic renal damage sets in, making evaluation of secondary pathology more difficult to assess. In that case, more accurate methodologies can be used to measure GFR by actually measuring serum clearance.

Frequently used tracers for assessing GFR are 99mTcdiethylenetriaminepentaacetic acid (Tc-99m DTPA) and I-125 Iothalamate since they are both highly filtered by the glomeruli, with minimal to no tubular secretion $[5,6]$. The former can be quantitatively assessed using imaging only after camera calibration with a syringe standard. This is the easiest technique to perform, can be done with all renal scans that use Tc-99m DTPA, but is also the least accurate methodology. Alternatively, both tracers can be used to assess GFR by getting serial blood draws following injecting the patient. The counts obtained in the aliquots can be converted into clearance, and directly relate to GFR. This methodology is much more labour intensive and can lead to errors if attention to details is not followed. ${ }^{125}$ I Iothalamate is more accurate for GFR assessment than Tc-99m DTPA, but it cannot be imaged. If additional information from the split function is available from imaging, then along with the absolute GFR calculations, the absolute GFR of each kidney can be inferred. Whereas this can all be done within a single study using Tc-99m DTPA, it is possible to do another study using one of the Tc-99m based imaging tracers to assess split function, and then using the ${ }^{125}$ I Iothalamate results to absolutely quantify each kidney's GFR. Additionally ${ }^{125} \mathrm{I}$ Iothalamate is significantly more expensive than all the other tracers.

\section{Quantitation in clinical molecular imaging}

From its earliest days, PET has had the inherent ability to quantify radiotracer uptake from the images due to its unique physics and 
dependence upon attenuation correction. Various models have been used to quantify uptake, with the greatest clinical impact being the development of the Standardized Uptake Values (SUVs) which are used in conjunction with F-18 Fluorodeoxyglucose (FDG) imaging in Oncology. This has been widely used in research papers, and is routinely quoted in clinical reports.

SUVs have numerous inherent limitations when looked at closely. There are many different types of SUVs (eg. SUV $\max , S U V_{\text {mean }}$, $S U V_{\text {peak }}$, etc.) each of which may be better utilized in certain scenarios, but by far the most commonly used clinically is the $\mathrm{SUV}_{\max }$ due to its highest inter-reader reproducibility. The SUV values have technical limitations, and vary greatly upon many factors, such as scan processing parameters, software versions, model of PET camera used, just to name a few [7]. SUVs correlate well with many malignancies and have shown to be significantly higher in values than inflammatory and other benign processes, but there is wide variability in the range of various physiologic processes, leading to significant overlap between benign and malignant uptake. Hence, SUVs cannot be used reliably to distinguish between the two without additional context from clinical or imaging information [8].

In theory, SUVs represent the degree of metabolic activity in a lesion, and have been shown to have both diagnostic and prognostic value, and at times may help guide management and therapies. Still, there has yet to be strong evidence that the use of SUVs in the assessment of most clinical scans has an incremental benefit in the diagnostic value and management potential as compared to an expert reader's qualitative evaluation of a scan. If not used in the appropriate context, the practical utility of absolute quantitation could be highly questionable, provide mostly superfluous information, if not at times even misleading.

Arguably, there could be very specific situations where SUVs may add value to a qualitative clinical assessment, such as monitoring for sarcomatous transformation of neurofibromatosis nodules, where serial SUV evaluation appears to improve diagnostic value over qualitative assessments. The lesions are intensely metabolically active, but some papers suggest that quantitation using a cutoff SUV, or calculating the Lesion/Liver uptake ratios, could be used to screen for malignant sarcomatous transformation. Nevertheless, the association is not very strong and is non-specific, so even though the incremental value of quantitation may be beneficial, it is still somewhat limited in scope [9].

When documenting clinical response to therapy in Lymphoma with FDG PET scans, the standard of care assessment is a primarily qualitative evaluation using the Deauville Scale, and not the SUV. The reason for this is the recognition of the vast number of inherent limitations of absolute quantitation with SUVs, but using qualitative approaches that also standardize how these scans are evaluated [10]. Furthermore, the vast majority of clinical information available on the utility of SUVs comes from the literature generated using FDG. With the clinical introduction of newer tracers, the established SUV values for FDG are meaningless in this setting, and have to be regenerated for each tracer. It is more likely that readers will learn evaluating qualitative images faster than waiting for clinical data to be generated which clearly demonstrates the incremental practical benefit of quantification for each tracer.

More recently with the introduction of immunotherapies, such as checkpoint inhibitors, the situation in evaluating therapy response has become even more complicated. Differentiating between progressive disease and a robust response to treatment at time points earlier than 6 months after therapy is an area of active research. After 3 months of initiating immunotherapy, FDG PET scans may demonstrate not only increased intensity of uptake, but also significantly increased size of the lesions and area involved, and may even show apparent new sites of disease. This 'flare' response qualitatively looks indistinguishable from a treatment failure, and better evaluated after at least another 3 months, when this response may have subsided. Newer areas of work are attempting to show that percentage changes in SUV may offer some predictive values of responses better than a qualitative analysis, but that is not optimal [11]. Overall treatment response or failure has to be assessed on both imaging and clinical information, or rely on newer tracers to be developed to evaluate this phenomenon.

\section{Quantification in theranostics}

Perhaps the penultimate goal is to be able to most accurately predict therapeutic success of a radionuclide therapy to patients, and more importantly, predict the dosage required pre-therapeutically to optimize therapeutic response. This has given rise to the idea of Theranostics, where a diagnostic image can at least qualitatively predict response to a therapy, and ideally absolute quantitation of uptake should correlate to degree of clinical response.

The first theranostic agent recognized is Radioiodine ${ }^{131} \mathrm{I}$, which can be imaged and provides therapeutic effects when delivered at higher dosages. Classically, the thyroid uptake has been used in conjunction with the diagnosis of Graves' disease, but also subsequently in the semi-qualitative assessment for determining treatment dosage with ${ }^{131} \mathrm{I}$ for this and other hyperactive thyroid diseases amenable to radioiodine therapy. Usually, the standard 24 hour uptake is most often used to calculate the dosage, but to most accurately assess adequate dose delivery, a formal dosimetry study should be performed. This is laborious and expensive to perform. Furthermore, accurate gland size is needed, which would require routine detailed imaging, as opposed to a clinical evaluation on physical examination, which is often the case. Even if the these variables are well defined, dose response varies with many other factors, such as radiosensitivity of the gland, relative hypoxic state, heterogeneity of uptake, etc. all of which can depend on the type of disease, length of involvement, amonst other factors. Thus, despite detailed calculations, the outcomes may still be quite variable, and so even routine gross estimation of size and uptake are felt to be adequate to predict clinical response within the known variability of responses. Given that this is benign disease and relatively lower therapeutic dosages are involved, the necessity for accuracy is less important.

In the case of well differentiated thyroid malignancies, adequate dose delivery is more of an issue. Still, most thyroid cancers tend to be relatively low risk and indolent, so exact dose delivery has little impact on long term outcomes. This becomes more pressing with higher risk disease, and where the disease starts to lose its ability to take up radioiodine. Some studies have shown the ability to calculate lesional dosimetry using ${ }^{124}$ I, a PET agent [12]. Even then, it is possible that accurate targeting of tumors may help destroy specific lesions, but without impacting overall survival since the same patient may have other disease which is less responsive to ${ }^{131} \mathrm{I}$ therapies.

More recently, there has been the development of hepatic malignancy therapies with Y-90 labeled microspheres Transarterial Radioembolization (TARE). The procedure involves doing a pretherapy scan after an injection of $99 \mathrm{mTc}$-macroaggregated albumin (MAA) to look at the distribution of tracer in the liver, and to assess 
for any extrahepatic shunts. Based on this, and calculations from anatomic radiographic imaging of the liver from which compartmental models can be created, a specific dosage of Y-90 microspheres is given to the patient to ablate hepatic lesions. The limitations of the compartmental models is that they are less likely to predict adequate dose delivery than patient specific methods [13]. They do not predict dose delivery, nor the onset of hepatic toxicity well. Tumour specific dosimetry has been shown to be able to predict this better in some small studies, including the use of PET-MR [14]. Ultimately, the real test is whether an approach that might better predict therapeutic response and limit toxicity also improves overall outcomes is yet unknown.

Like thyroid malignancies, there are many clinical variables that would go into predicting response, so absolute quantitation of dosimetry would provide only a portion of the solution. This overall basic problem persists in various other radionuclide therapies as well. Hence, many of the new emerging therapies use dosages which are either fixed empirical amounts, or adjusted to simpler parameters such as Body Weight, etc.

\section{Quantification in cardiac nuclear medicine}

An area where quantitation has had the greatest impact is in Cardiac Nuclear Imaging. Since the early days of Thallium $\left({ }^{201} \mathrm{Tl}\right)$ imaging to the Tc-99m labeled perfusion agents (Sestamibi and Tetrofosmin), qualitative and semi-quantitative imaging in the field has proven to be very useful. Relative quantitation of perfusion defects, whether they were fixed or reversible, has shown to carry strong prognostic indicators, and thus guides managements. These were eventually standardized by using cardiac mapping systems and normals database. Further added to this was the ability to perform gated SPECT, which allowed evaluation of Ejection Fraction with the perfusion component, which not only improved the accuracy of the qualitative assessment of the perfusion component, but also provided an independent prognostic significance to the test results. When evaluating viability, percent changes in myocardial uptake with ${ }^{201} \mathrm{Tl}$ had good correlation with hibernating and identifying salvageable - myocardium. Some of these concepts translated into viability with PET as well. Viability imaging did fall short as a routine clinical procedure based on outcomes in the STICH (Surgical Treatment for Ischemic Heart failure) trial, but is still occasionally used as an adjunct to make difficult clinical decisions [15]. Nevertheless, these approaches have had significant limitations, such as decreased sensitivity in the detection of multivessel disease, or in certain cases, tight stenoses.

Eventually, PET imaging became more accessible as Oncologic indications made it more widely available, and with the increased presence of PET Cardiac tracers, allowed Cardiac PET to become a feasible modality. The advantage of PET is several fold. It has higher spatial resolution than SPECT, and inherent attenuation correction, which is also superior to SPECT-CT. Additionally, PET tracer kinetics tend to be better than the SPECT agents, especially the currently available Tc-99m labeled agents, increasing accuracy for disease assessment. An important aspect of PET is its ability to do absolute quantitation. Even with PET, it is well known that qualitative and semiquantitative imaging can miss significant disease, but by adding quantitative analysis of Myocardial Blood Flow (MBF) and calculating Coronary Flow Reserve (CFR), the diagnostic capability increases significantly, including carrying independent prognostic significance $[16,17]$. The concept has been further modified to evaluating Coronary Flow Capacity (CFC), which essentially relies on the quantitation of flow at rest and stress, and reduces some of the variability of measuring CFR alone [18]. The calculations do require modeling of the relevant tracers. Despite that, there are still some limitations as different software may give varying results, which can be significant in terms of diagnosing patients as being normal vs. Abnormal [19]. Research is ongoing to try and standardize these entities to ensure more clinically accurate results.

\section{Emerging horizons}

Quantitation is gaining increasing attention as a way to enhance current qualitative and quantitative diagnostic imaging. This is partly due to improvements in technology, newer software, as well as better modeling of known tracer physiology into the calculations. Given the popularity with PET quantitation, there has naturally been interest in being able to similarly quantitate with SPECT, and in particular SPECT-CT. There have been several hurdles to this point in time since the physics of SPECT imaging have limitations, making absolute quantitation much more difficult. The resolution of SPECT is simply lower than PET, and thus limiting its accuracy of its measurement. Additional corrections for attenuation and scatter are also not as robust as PET. Still, there has been a steady development in this field to the point where quantitative SPECT appears to be rising to the surface and potentially could have a clinical impact [20] New software and technologic developments that are now clinically available have begun to show some ability to provide absolute quantitation. Most SPECT imaging has diagnostic criteria that has been used for a long time, and it has yet to be studied whether there will be a definite incremental benefit of adding quantitation to routine qualitative evaluations, especially given the ultimately recognized failure of SUVs to do so routinely in the PET side. Nevertheless, this continues to be an ongoing area of study that will be looked at critically.

The inherent limitations of SPECT imaging have started being addressed more recently with newer sophisticated tools that allow for improvements in corrections. Although still not as accurate as PET, with the appropriate corrections the system can still be made to provide with accurate count statistics in a more reliable fashion, which would in turn allow this new tool to be implemented clinically and evaluate its potential utility as an enhanced diagnostic tool [21].

There have been many developments to make quantitation feasible in a routine fashion. Firstly, it's important to recognize that no matter what system is being used, whether SPECT, PET, or even planar imaging-quantitation has to be standardized and calibrated against known entities, and then use the device accurately to count the desired object. Even then, the standard itself should be conducive to allowing for accurate measurements. The flangeless Esser phantomTM can not only assess qualitative imaging, but is also specifically suited for calibration for virtual quantitation due to its differences in its design compared to traditional phantoms [22].

There is also increased interest from many software vendors to develop products that can handle quantitative data better, beyond the traditional tools. These rely on newer processing methods which increase the possibility to calculate absolute quantification with more accurate data. With these technical tools coming up, the tougher issues with quantitative imaging can be tackled more concertedly. Initial attempts to look at some of the difficult problems outlined earlier have been attempted, and longer term data will be needed to see if they provide incrementally increased clinical value over current imaging results [23]. 
A development relying more heavily on quantitation as a primary diagnostic tool over the traditional qualitative imaging is the Fleming method for tissue and vascular differentiation and metabolism $\left(F\right.$ TVDM $\left.^{\odot \odot}\right)$ and the Fleming Harrington redistribution wash-in washout (FHRWW), which develop old ideas and incorporate many of the methods outlined earlier. These patented procedures require appropriate camera calibration, tracer modeling and image based quantitation, and are used to manage coronary disease [24].

When FMTVDM is used with breast enhanced scintigraphy testing (BEST) and Sestamibi, it enhances scintigraphic detection of breast cancer. Sestamibi scintigraphy in breast imaging is not new, and is also known as Breast Specific Gamma Imaging (BSGI) or scintimammography. It was used as a tumour imaging agent just prior to the astronomic rise in popularity of FDG PET imaging. Sestamibi found a niche with BSGI, which although superior to mammography, did not gain traction in part because of the competing modalities of breast ultrasound and Magnetic Resonance Imaging (MRI). One of the limitations to date has been the ability to distinguish between normal, inflamed and malignant tissues. FMTVDM-BEST uses Sestamibi's kinetics, along with proprietary patented calculations, to significantly enhance BSGI, improving its ability to distinguish between the various types of tissues and enhance accuracy of the diagnosis of breast cancer $[25,26]$.

With the advent of new cardiac specific cameras and the success of PET, there has naturally been an interest in developing models for measuring CFR with SPECT. Although studies in this area are ongoing, some initial results appear promising [27]. In this regards, FMTVDMFHRWW have used patented methods to calculate tracer redistribution, and demonstrating that they can predict stenoses using quantification that with routine qualitative imaging would have missed the lesions. The methodology can be extended to different tracers, and can thus be used with either PET or SPECT, and can even be technically adapted for use with probes. It is also independent of the type of stress the patient undergoes.

Of additional benefit of the FMTVDM-FHRWW methodology is that it actually analyzes and detects the presence of coronary disease based on an early and delayed image based on the stress images only, and thus do not require the conventional rest images. This goes along with the current trend to do stress only images and thus decrease radiation exposure to the patient. Several studies have shown that the negative predictive value and subsequent outcome of stress only images is good for most patients, except for a subset of higher risk patients [28]. The FMTVDM-FHRWW method can provide additional information that could help detect these subset of patients. In a VA and another multinational study, the FMTVDM-FHRWW was able to detect tight stenoses in patients in whom conventional imaging did not detect the lesions [29]. Moreover, the FMTVDM-FHRWW method may be a paradigm shift as it does not simply detect disease as a binary answer, but rather regards it as a continuum, and thus the results correlate well to the degree of stenoses and physiologic perfusion derangement as compared to coronary angiography $[30,31]$. Thus, the treating physician is provided with important additional clinical information that can help manage the patient given a better understanding of the disease, which is a goal of current personalized and precision medicine (Figure 1).

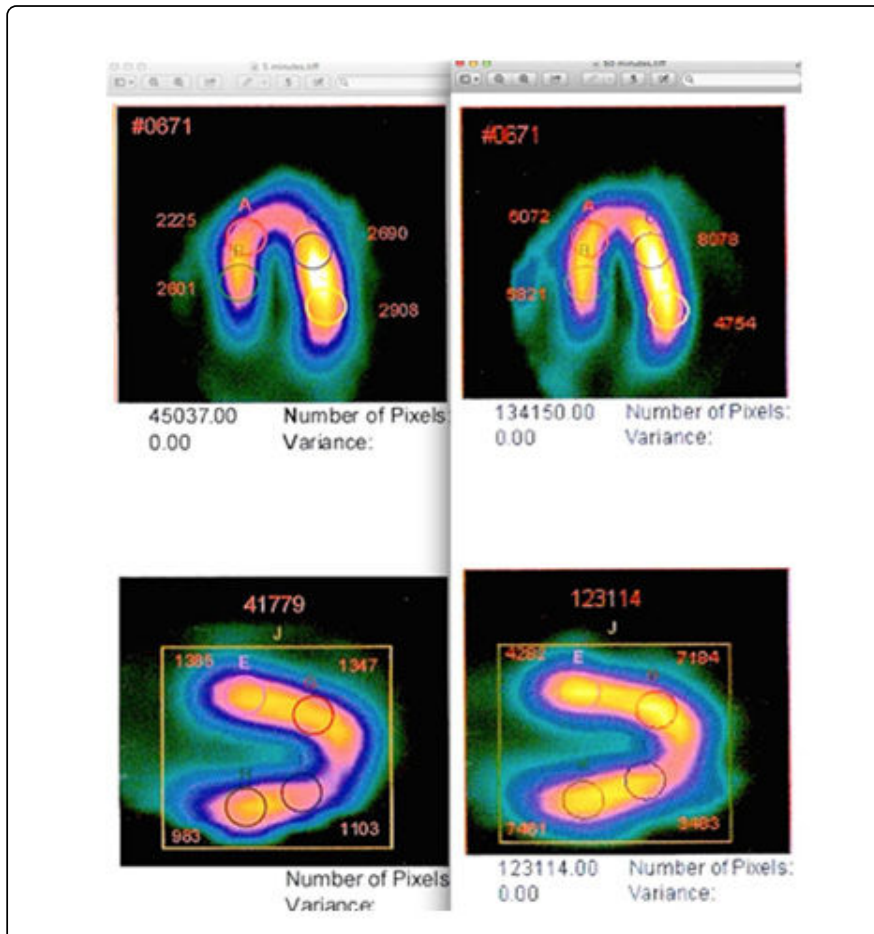

Figure 1: FMTVDM-FHRWW (Cardiac protocol) ${ }^{\odot}{ }^{\circledR}$. Application of TRUE QUANTIFICATION following isotope redistribution. Image displays in horizontal (top) and vertical (bottom) long axis views show TRUE QUANTIFICATION using measurement of Sestamibi redistribution using FMTVDM $^{\odot \odot}$. While each reconstructed image revealed "qualitatively" normal appearing MPI, the TRUE QUANTIFICATION measurement showed lower Sestamibi counts in each myocardial region at 5-minutes (left panels) compared with the 60-minute acquisitions demonstrating "wash-in" seen with vulnerable inflammatory plaques and critically narrowed arteries. This TRUE (not virtual) QUANTIFICATION demonstrated triple vessel coronary artery disease in this individual. Courtesy, Dr. RM Fleming, MD FHHIOmnificImaging-Camelot, USA.

\section{Conclusion}

Quantitative analysis has been present Nuclear Medicine from its inception. Although there are various areas where quantification was developed, the vast majority of Nuclear Imaging utilized qualitative evaluation for diagnoses. The clinical value of quantification has been mixed. In routine scans, such as Renal Scintigraphy, or calculating CFR in Cardiac PET, the value of quantification has been significant in adding to the diagnosis provided by qualitative imaging. In Oncologic PET, the SUV is widely used, but for most cases there is little incremental benefit over qualitative imaging in the hands of expert readers. Additionally, newer tracers are currently approached qualitatively, with the value of quantitation still being studied. With renewed interest in quantitation, vendors are producing software that can provide such information. Newer phantom designs will also allow cameras to be better calibrated for quantitation. The development of the FMTVDM-FHRWW and BEST imaging methods could represent a part of a paradigm shift in cardiac nuclear imaging, and is following the trend of providing overall risk stratification of patients with 
Nuclear Medicine, rather than a simple binary diagnostic answer from medical imaging. It significantly increases the detection rate of coronary disease, as well as malignancy detection in breast imaging. Moreover, it is a quantitative method that views disease as a linear process, and correlates well with degree of disease. Hence, it is clear that the era of absolute quantitative imaging is beginning, and that there is already information that provides significant clinical impact. The data is suggestive that these approaches will impact patient care and may be cost effective, but it remains to be seen whether the advantages of doing quantitative imaging will provide enough of an incremental benefit that they will be adopted as a part of the new standard of care.

\section{References}

1. Piai DB, Quagliatto R, Toro I, Cunha NC, Etchbehere E, et al. (2004) The use of SPECT in preoperative assessment of patients with lung cancer. Eur Respir J 24: 258-262

2. Takenaka D, Ohno Y, Koyama H, Nogami M, Onishi Y, et al. (2010) Coregistered perfusion SPECT/CT: Utility for prediction of improved postoperative outcome in lung volume reduction surgery candidates. Eur J Radiol 74: 465-472

3. Piepsz A, Dobbeleir A, Erbsmann F (1977) Measurement of separate kidney clearance by means of $99 \mathrm{mTc}$-DTPA complex and a scintillation camera. Eur J Nuc Med 2: I73-177

4. Gates GF (1982) Glomerular filtration rate: Estimation from fractional renal accumulation of 99mTc-DTPA." Am J Roenlgenol 138: 565-570.

5. Hilson AJW, Mistry RD, Maisey MN (1976) Technetium-99m-DTPA for the measurement of glomerular filtration rate. Br J Rad 49: 794-796.

6. Wiener SN, Shah YP, Mares RM, Flynn MJ (1982) Correlation of I-125 Iothalamate and Tc-99m DTPA measurements of GFR using the single injection method. Clin Nucl Med 7: 359-363.

7. Schwartz J, Humm JL, Gonen M, Kalaigian H, Schoder H, et al. (2011) Repeatability of SUV measurements in serial PET. Med Phys 38: 2629-2638.

8. Chao-Jung Chen, Bi-Fang Lee, Wei-Jen Yao, Lili Cheng, Pei-Shan Wu, et al. (2008) Dual-Phase 18F-FDG PET in the diagnosis of pulmonary nodules with an initial Standard Uptake Value Less Than 2.5. AJR 191: 475-479

9. Combemale P, Valeyrie-Allanore L, Giammarile F (2014) Utility of 18FFDG PET with a Semi-Quantitative Index in the Detection of Sarcomatous Transformation in Patients with Neurofibromatosis Type 1. PLoS One 9: e85954.

10. Meignan M, Gallamini A, Haioun C (2009) Report on the First International Workshop on interim-PET scan in lymphoma. Leukemia \& Lymphoma 50: 1257-1260

11. Rossi S, Toschi L, Castello A, Grizzi F, Mansi L, et al. (2017) Clinical characteristics of patient selection and imaging predictors of outcome in solid tumors treated with checkpoint-inhibitors. Eur J Nucl Med Mol Imaging 44: 2310-2325

12. Erdi YE, Macapinlac H, Larson SM, Erdi AK, Yeung H, et al. (1999) Radiation Dose Assessment for I-131 Therapy of Thyroid Cancer Using I-124 PET Imaging. Clin Pos Imag 2: 41-46

13. Kafrouni M, Allimant C, Fourcade M (2018) Retrospective voxel-based dosimetry for assessing the ability of the body-surface-area model to predict delivered dose and radioembolization outcome. J Nucl Med 59: 1289-1295

14. Fowler KJ, Maughan NM, Laforest R, Saad NE, Sharma A, et al. (2016) PET/MRI of hepatic 90Y microsphere deposition determines individual tumor response. Cardiovasc Intervent Radiol 39: 855-864
15. Robert O. Bonow, Gerald Maurer, Kerry L. Lee, Thomas A. Holly, Philip F. Binkley, et al. (2011) Myocardial viability and survival in ischemic left ventricular dysfunction. N Engl J Med 364: 1617-1625.

16. McArdle B, Dowsley TF, Cocker MS, Ohira H, deKemp RA, et al. (2013) Cardiac PET: Metabolic and functional imaging of the myocardium. Semin NuclMed 43: 434-448.

17. Tio RA, Dabeshlim A, Siebelink HM (2009) Comparison between the prognostic value of left ventricular function and myocardial perfusion reserve in patients with ischemic heart disease. J Nucl Med 50: 214-219

18. Van de Hoef TP, Echavarría-Pinto M, Escaned J, Piek JJ (2017) Coronary flow capacity: concept, promises, and challenges. Int J Cardiovasc Imaging 33: 1033-1039

19. Nesterov, Lee, Moody (2016) The Status and Future of PET Myocardial Blood Flow Quantification Software. Ann Nucl Cardiol 2: 106-110

20. Ljungberg (2018) Absolute quantitation of SPECT studies. Semin Nucl Med 48: 348-358

21. Kangasmaa TS, Constable C, Hippeläinen E, Sohlberg AO (2016) Multicenter evaluation of single photon emission computed tomography with third-party reconstruction software. Nucl Med Commun 37: 983-987

22. Jenkins L, Ross E, Sohlberg A, Esser PD (2016) A new SUV phantom for SPECT and PET. J Nucl Med 57: S2 592

23. Hippeläinen E, Tenhunen M, Mäenpää H, Sohlberg A (2016) Quantitative accuracy of $177 \mathrm{Lu}$ SPECT reconstruction using different compensation methods: Phantom and patient studies. EJNMMI Res 6: 16

24. Fleming RM, Harrington GM, Baqir R (2010) Renewed application of an old method improves detection of coronary ischemia: A higher standard of care. Fed Pract 27: 22

25. Fleming RM, Dooley WC (2002) Breast enhanced scintigraphy testing distinguishes between normal, inflammatory breast changes, and breast cancer: A prospective analysis and comparison with mammography. Integr Cancer Ther 1: 238-245

26. Fleming RM (2002) Mitochondrial uptake of sestamibi distinguishes between normal, inflammatory breast changes, pre-cancers, and infiltrating breast cancer. Integr Cancer Ther 1: 229-237

27. Storto G, Cirillo P, Vicario MLE (2004) Estimation of coronary flow reserve by Tc-99m sestamibi imaging in patients with coronary artery disease: Comparison with the results of intracoronary Doppler technique. J Nucl Cardiol 11: 682-628.

28. Einstein AJ, Johnson LL, DeLuca AJ (2015) Radiation dose and prognosis of ultra-low-dose stress-first myocardial perfusion SPECT in patients with chest pain using a high-efficiency camera. J Nucl Med 56: 545-551

29. Fleming RM, Fleming B, McKusick A (2018) USVAH study demonstrates statistically significant improvement in diagnosis and care of U.S veterans using FMTVDM-FHRWW@ (P) 'quantitative' nuclear imaging. The era of truly quantitative stress-first, stress-only imaging has begun. J Nucl Med Radiat Ther S9: 006

30. Fleming RM, Fleming MR, McKusick A, Chaudhuri TK (2018) Multi center clinical trial confirms FMTVDM@ $\odot$ MPI in seven modern clinical laboratories in the USA and Asia. Artificial Intelligence (AI) with True Quantification. J Nucl Med Radiat Ther 9: 4

31. Fleming RM, Fleming MR, Chaudhuri T, McKusick, A, Dooley WC, et al. (2018) Both percent diameter stenosis (\%DS) and coronary flow reserve can be derived directly from myocardial perfusion imaging using FMTVDM and measurement of isotope redistribution. J Nucl Med Radiat Ther 9: 1 